\title{
Estudo comparativo entre o Doppler Ultra-Som e a arteriografia convencional no diagnóstico das obstruções das artérias digitais no membro torácico do eqüino (Equus caballus)
}

\section{A comparative study between Doppler Ultrasound and conventional artheriography to identify digital arterial obstruction of thoracic limb in horse (Equus caballus)} \author{
Leny Pereira Sant'Anna Junior, ${ }^{\star}$ Daniel Augusto Barroso Lessa, ${ }^{\star \star}$ Eugênio Oliveira Henriques,
Antônio Carlos da Silva
}

\section{Resumo}

\begin{abstract}
A presente pesquisa objetivou comparar os traçados do Doppler Ultra-Som (método não-invasivo) e as imagens da arteriografia convencional (método invasivo padrão) frente ao diagnóstico das obstruções das artérias digitais do membro torácico no eqüino. Utilizou-se um aparelho de Doppler Ultra-Som bidirecional de ondas contínuas para examinar a artéria digital palmar comum II, a artéria digital palmar própria medial e a artéria digital palmar própria lateral de 11 eqüinos de equitação, sem raça método torácicos, obtendo-se 66 arteriografias, onção. Subseqüentemente realizaram-se 22 exames arteriográficos nos membros

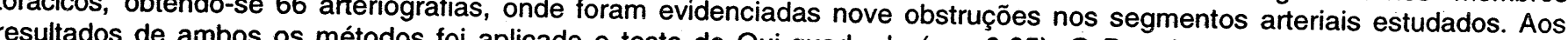
resultados de ambos os métodos foi aplicado o teste de Qui-quadrado $(p<0,05)$. O Doppler Ultra-Som demonstrou uma sensibilidade de $77,7 \%$ e uma especificidade de $100 \%$ quando comparado à arteriografia. Conclui-se que o método do Doppler Ultra-Som é valioso no diagnóstico das obstruçōes das artérias digitais dos membros torácicos no cavalo, principalmente quando se quer evitar métodos invasivos de diagnóstico (arteriografia).
\end{abstract}

Palavras-chave: eqüino; Doppler Ultra-Som; arteriografia.

\begin{abstract}
This research develop a comparative study between the records of Doppler Ultrasound (noninvasive method) and the images of conventional artheriography (invasive method gold-standard) to identify obstructions in the digital arteries of thoracic limb in horse. A bidirectional continuous wave Doppler Ultrasound was used to investigate the palmar commom digital artery, lateral palmar digital artery and medial palmar digital artery. Eleven mixed breed horses with ages between 16 and 24 years were used. examination, the artheriograms revealed 9 with the Doppler Ultrasound identifying 7 obstructions. After 22 artheriographical by Chi-Square test of digital arterial obstruction when compared with of digital arteries in horses thoracic limber value of the Doppler Ultrasound to diagnose obstructions
\end{abstract}

Keywords: horse; Doppler Ultrasound; artheriography.

\section{Introdução}

Técnicas arteriográficas e investigações anátomo-radiológicas das artérias digitais nos eqüinos foram descritas por Coffman et al. (1970), Hertsch (1973), Ackerman et al. (1975),
Fricker et al. (1982), Stanek et al. (1982), Botti (1983), Litzke (1986), Silva (1988, 1995), Lessa (1993) e Lessa et al. (1997). Estes métodos invasivos, apesar de permitirem a visualização da morfologia arterial e serem definitivos na identificação tanto do local como da extensão topográfica de arteriopatias

\footnotetext{
* Médico Veterinário Autônomo, Rua Borda do Mato, 106/104, CEP 20561-200, Rio de Janeiro, RJ, Brasil.

* Instituto de Veterinária, Universidade Federal Rural do Rio de Janeiro.

*** Médico Veterinário, capitão da PMRJ.

**** Instituto Biomédico, Universidade Federal Fluminense.
} 
obstrutivas, não permitem obter informações relacionadas com a hemodinâmica local, além de requererem contenção farmacológica e certo risco para o paciente.

Para o estudo vascular, o exame Doppler Ultra-sonográfico (não-invasivo) é utilizado na medicina tanto como técnica preliminar diagnóstica para a identificação de obstruções arteriais, como também permite detectar alterações hemodinâmicas em artérias que apresentam velocidades de fluxo modificado (Panico, 1995).

Muito embora os resultados de exames vasculares nãoinvasivos sejam comparados com a angiografia para verificar sua confiabilidade, é necessário determinar a sensibilidade e a especificidade dos procedimentos não-invasivos para testar sua validade como técnica diagnóstica (Bellen, 1987).

\section{Material e métodos}

\section{Animais}

Foram utilizados 11 eqüinos de equitação sem raça definida, sendo dez machos castrados e uma fêmea, claudicantes e não-claudicantes, com idade entre 16 e 24 anos, pesando de 330 a $450 \mathrm{~kg}$, pertencentes ao Regimento Escola de Cavalaria (REsC), Rio de Janeiro, Ministério do Exército (Tabeia 1).

Tabela 1: Identificação dos animais e das extremidades dos apêndices locomotores dos membros torácicos

\begin{tabular}{rrrrrr}
\hline Caso & $\begin{array}{c}N^{2} \text { do } \\
\text { registro }\end{array}$ & $\begin{array}{c}\text { Peso } \\
(\mathbf{k g})\end{array}$ & Sexo & $\begin{array}{c}\text { Idade } \\
\text { (anos) }\end{array}$ & Membro \\
\hline 1 & 0264 & 360 & $\mathrm{M}$ & 17 & TDC \\
2 & 0264 & 360 & $\mathrm{M}$ & 17 & TENC \\
3 & 0054 & 330 & $\mathrm{M}$ & 18 & TDNC \\
4 & 0054 & 330 & $\mathrm{M}$ & 18 & TEC \\
5 & 0076 & 340 & $\mathrm{M}$ & 22 & TDNC \\
6 & 0076 & 340 & $\mathrm{M}$ & 22 & TENC \\
7 & 1905 & 422 & $\mathrm{M}$ & 16 & TDC \\
8 & 1905 & 422 & $\mathrm{M}$ & 16 & TEC \\
9 & 0002 & 380 & $\mathrm{M}$ & 22 & TDNC \\
10 & 0002 & 380 & $\mathrm{M}$ & 22 & TENC \\
11 & 0349 & 350 & $\mathrm{M}$ & 19 & TDC \\
12 & 0349 & 350 & $\mathrm{M}$ & 19 & TEC \\
13 & 0189 & 361 & $\mathrm{~F}$ & 21 & TDNC \\
14 & 0189 & 361 & $\mathrm{~F}$ & 21 & TENC \\
15 & 0060 & 400 & $\mathrm{M}$ & 23 & TDC \\
16 & 0060 & 400 & $\mathrm{M}$ & 23 & TENC \\
17 & 0092 & 370 & $\mathrm{M}$ & 24 & TDNC \\
18 & 0092 & 370 & $\mathrm{M}$ & 24 & TENC \\
19 & 3234 & 347 & $\mathrm{M}$ & 22 & TDNC \\
20 & 3234 & 347 & $\mathrm{M}$ & 22 & TENC \\
21 & 3108 & 400 & $\mathrm{M}$ & 19 & TDNC \\
\hline 2 & 3108 & 400 & $\mathrm{M}$ & 19 & TENC \\
\hline
\end{tabular}

$M=$ macho; $F=$ fêmea; $T D C=$ torácico direito claudicante; TDNC = torácico direito não-claudicante; TEC = torácico esquerdo claudicante; TENC = torácico esquerdo não-claudicante.

\section{Exame Doppler Ultra-Som}

A preparação do animal constituiu-se na realização do desferrageamento do membro torácico, da higienização de toda região distal ao carpo com sabão neutro, da tricotomia da região metacárpica medial e das regiões lateral e medial da articulação metacarpofalangiana. $O$ animal foi colocado em um brete de manejo, à sombra e em temperatura ambiente durante 10 a 15 minutos para estabilização dos parâmetros fisiológicos.

Após o exame clínico geral, foi utilizado um aparelho Doppler Ultra-Som bidirecional ${ }^{1}$ com detector de velocidades positivas e negativas a partir do zero e com capacidade para registro em um canal, para a obtenção dos resultados deste método.

A tecla de intensidade de ruídos arteriais do aparelho foi colocada na posição 5 e as teclas de registro gráfico ajustadas para a velocidade de $25 \mathrm{~mm} / \mathrm{s}$. Para o tamanho da onda foi selecionado $0 n^{\circ} 3$, o controle de gravação na posição bidirecional e o filtro de $3,5 \mathrm{~Hz}$.

Na região do sulco metacárpico, o transdutor de $8,0 \mathrm{MHz}$ do Doppler foi posicionado sobre a pele, formando, obliquamente com esta, um ângulo de inclinação de 45 a 60 graus com o plano sagital do membro, com a extremidade dos cristais voltadas cranialmente, utilizando um gel acústico ${ }^{2}$ para conexão da pele com o transdutor. A identificação dos ruídos arteriais pelo transdutor pôde ser auxiliado pela palpação prévia do pulso da artéria digital palmar comum II e pelo ruído da veia adjacente (veia palmar medial). Quando o fluxo estava presente, foi possível obter o sinal audivel e os registros das ondas de velocidade do segmento distal da artéria digital palmar comum II (Figura 1). Posteriormente o transdutor foi posicionado na face lateral e medial da articulação metacarpofalangiana na região abaxial dos sesamóides proximais, novamente com os cristais voltados cranialmente e com ângulo de 45 a 60 graus, para obter os ruídos e os registros gráficos da artéria digital palmar própria lateral e da artéria digital palmar própria medial, respectivamente.

Os resultados dos registros de cada segmento arterial examinado foram julgados segundo duas maneiras distintas: ser negativo (ausência da obstrução arterial), caracterizados pela existência de ondas de velocidade no traçado (Figura 1), ou, na segunda hipótese, ser positivo Os resultados dos registros de cada segmento arterial examinado foram julgados segundo duas maneiras distintas: ser negativo (ausência da

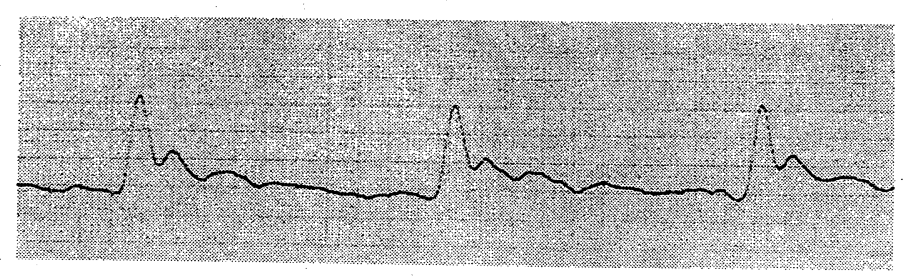

Figura 1: Registro termográfico de fluxo arterial pelo Doppler Ultra-Som a $25 \mathrm{~mm} / \mathrm{s}$.

\footnotetext{
1 Doppler Ultra-Som, Modelo 1052-C, Vascular Mini-Lab Parks Medical Eletronics, Inc.).

2 Aquasonic 100 (Parks Medical Eletronics, Inc.).
} 
obstrução arterial), caracterizados pela existência de ondas de velocidade no traçado (Figura 1), ou, na segunda hipótese, ser positivo (presença de obstrução), caracterizados pela presença de uma linha isoelétrica no traçado (Figura 2). Este critério de julgamento foi estabelecido antes da obtenção das imagens arteriográficas.

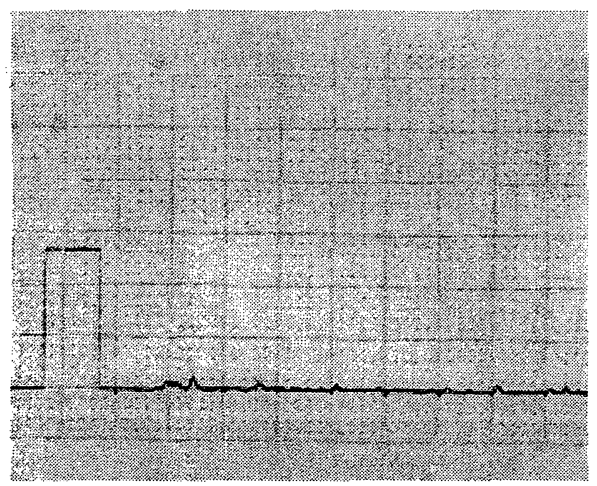

Figura 2: Registro termográfico de obstrução ao Doppler Ultra-Som. Aparelho ajustado para velocidade de $25 \mathrm{~mm} / \mathrm{s}$.

\section{Exame arteriográfico}

Foram realizados 22 exames arteriográficos palmares nos dois membros torácicos de 11 eqüinos, através da punção e cateterização da artéria digital palmar comum II, com o animal em estação segundo a técnica de Henriques (1998).

As arteriografias dos segmentos arteriais estudados foram obtidas utilizando como meio de contraste o ioxaglato de meglumina e sódio, ${ }^{3}$ filmes de fluorescência verde ${ }^{4}$ montados sob chassis com écran fluorescente ${ }^{5}$ acoplada nas medidas $30 \mathrm{~cm} \times 40 \mathrm{~cm}$ e uma régua escanográfica de acrílico de $40 \mathrm{~cm} .{ }^{6}$ Foram realizadas nas posiçōes dorso-palmar (DPa), latero-medial (LM) e dorso-lateral oblíqua pálmaro-medial obliqua (D45ㄴ-PaMO). Foi empregado um aparelho de raios $X$ portátil com capacidade de $25 \mathrm{~mA}$ e $85 \mathrm{kV},{ }^{7}$ utilizando como técnica radiográfica: $80 \mathrm{kV}$ e $15 \mathrm{mAs}$, a uma distância foco filme de $80 \mathrm{~cm}$.

A leitura das arteriografias foi feita considerando a presença ou ausência de obstrução arterial, segundo os critérios descritos por Silva (1988).

\section{Tratamento estatístico}

Foi aplicado o teste não-paramétrico de $\chi^{2}$ (Qui-quadrado). Adotou-se o nivel de significância de $5 \%$ (Campos, 1983).

Foram determinadas as percentagens de sensibilidade e especificidade para verificar o método Doppler em relação ao método padrão (arteriografia), bem como a incidência de falsos-positivos e de falsos-negativos do Doppler Ultra-Som.

\footnotetext{
3 Hexabrix 320, Guerbet Produtos Radiológicos Ltda.

${ }^{4}$ Filmes para Raios X, 3M, Argentina, SACIFIA.

${ }^{5}$ Kodak Brasileira Comércio e Indústria Ltda:

- Casa do Radiologista, Rio de Janeiro, RJ.

${ }^{7}$ Aparelho de Raios X, FNX Manutenção de Aparelhos Médicos Ltda.
}

\section{Resultados e discussão}

Após a obtenção de 66 arteriografias e 66 exames Doppler Ultra-sonográficos das artérias digitais nos membros torácicos dos 11 eqüinos, perfazendo um total de 22 membros estudados, foi possivel alcançar os seguintes resultados:

\section{Arteriográficos}

Foram encontradas nove obstruções segmentares das artérias digitais, com a seguinte distribuição: uma na artéria digital palmar comum II no caso no 3 , sete na artéria digital palmar própria medial nos casos ne 1, 2, 12, 16 (Figura 3), 19 (Figura 4), 20 e 21, e uma na artéria digital palmar própria lateral no caso ne 17 (Tabela 2). Nos demais casos não foram observadas obstruções nestes segmentos arteriais (Figuras 5 e 6).

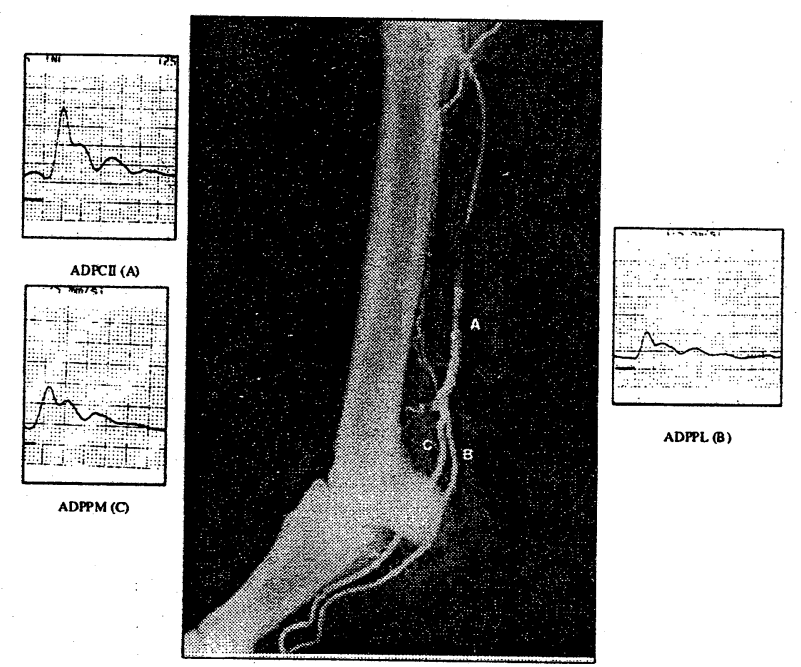

Figura 3: Arteriografia e Doppler Ultra-Som do caso 16. A arteriografia foi obtida na posição dorso-palmar (DPa) e os registros do Doppler UltraSom nos locais indicados pelas letras. $\mathbf{A}=$ artéria digital palmar comum II; B = artéria digital palmar própria lateral. Observa-se obstrução da artéria digital palmar própria medial (C), a nível da articulação metacarpofalangiana; apresentando traçado com linha isoelétrica correspondente. Nota-se a reabitação do segmento distal desta artéria através da circulação colateral (seta).

A incidência maior de obstruções completas na artéria digital palmar própria medial, $(77,7 \%)$, estão em conformidade com os relatos de Silva (1988), que utilizou animais pertencentes à mesma população do nosso estudo, onde das 12 obstruções das artérias do dígito relatadas, sete $(58,3 \%)$ se situavam na artéria digital palmar própria medial a nível da articulação metacarpofalangiana e de Fricker et al. (1982), onde todas as oito (100\%) obstruções que encontrou foram na artéria digital palmar própria medial. Silva (1998), embora não discuta a etiologia deste fenômeno, justifica a cateterização da artéria digital palmar própria lateral devido a alta incidência de obstruções na artéria digital palmar própria medial. Fricker et al. (1982) cita a denominação de endarterite obliterante, atribuída por Köhler (1950) para referir-se às obstruções arteriais digitais encontradas em seu estudo. Atribui a ocorrência maior de obstruções na artéria digital palmar própria medial a uma assimetria na regiāo da ramificação da artéria digital palmar comum II, que só ocorre no membro 


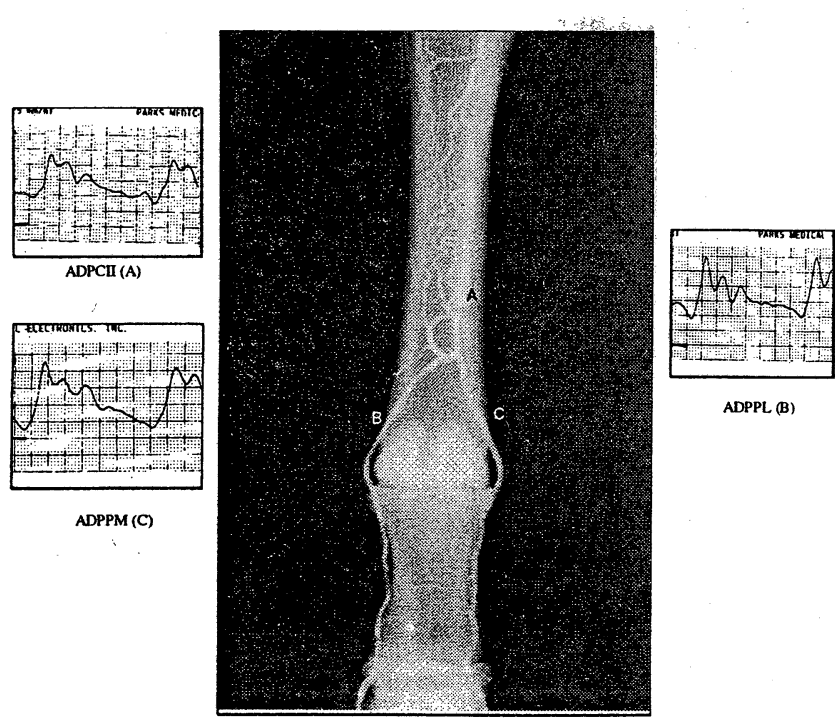

Figura 4: Arteriografia e Doppler Ultra-Som do caso 19. A arteriografia foi obtida na posição dorso-palmar (DPa) e os registros do Doppler Ultra-Som nos locais indicados pelas letras. $A=$ artéria digital palmar comum II; B = artéria digital palmar própria lateral. Observa-se obstrução da artéria digital palmar própria medial (C), a nível da articulação metacarpofalangiana, apresentando traçado com linha isoelétrica correspondente. Nota-se a reabitação do segmento distal desta artéria através da circulação colateral (seta).

Tabela 2: Distribuição e características arteriográficas das obstruções

\begin{tabular}{cccccc}
\hline Caso & $\begin{array}{c}\text { Artéria } \\
\text { obstruída }\end{array}$ & $\begin{array}{c}\text { Extensão } \\
(\mathrm{cm})\end{array}$ & Apresentação & $\begin{array}{c}\text { Circulação } \\
\text { Colateral }\end{array}$ & Membro \\
\hline 1 & ADPPM & 4,5 & $\mathrm{PL}$ & $\mathrm{C}$ & TDC \\
2 & ADPPM & 4.5 & $\mathrm{ST}$ & $\mathrm{C}$ & TENC \\
3 & ADPC II & 6,0 & $\mathrm{ST}$ & $\mathrm{N}$ & TDNC \\
12 & ADPPM & 8,0 & $\mathrm{PL}$ & $\mathrm{N}$ & TEC \\
16 & ADPPM & 3,5 & $\mathrm{ST}$ & $\mathrm{NC}$ & TENC \\
17 & ADPPL & 3,0 & $\mathrm{PL}$ & $\mathrm{C}$ & TDNC \\
19 & ADPPM & 10,0 & $\mathrm{PL}$ & $\mathrm{NC}$ & TDNC \\
20 & ADPPM & 3,0 & $\mathrm{PL}$ & $\mathrm{C}$ & TENC \\
21 & ADPPM & 18,0 & $\mathrm{PL}$ & $\mathrm{C}$ & TDNC \\
\hline
\end{tabular}

ADPC $\|$ = artéria digital palmar comum $\| ;$ ADPPM = artéria digital palmar própria medial; ADPPL = artéria digital palmar própria lateral; TDC = torácico direito claudicante; TDNC = torácico direito nãoclaudicante; TEC = torácico esquerdo claudicante; TENC = torácico esquerdo não-claudicante; $\mathrm{ST}=$ secção transversa; $\mathrm{PL}=$ ponta de lápis; $\mathrm{C}=$ confluente; $\mathrm{N}=$ normal; $\mathrm{NC}=$ não confluente.

torácico, ocasionando um trauma maior na região medial do osso sesamóide proximal medial, pois esta região coincide com o trajeto da artéria digital palmar própria medial que cruza o ligamento do osso sesamóide proximal medial. Este autor também cita Dunlop e Santos (1957) comparando etiologicamente estas obstruções com a obstrução da artéria femoral superficial no canal de Hunter que ocorre em humanos, também atribuída a trauma local devido a movimentos

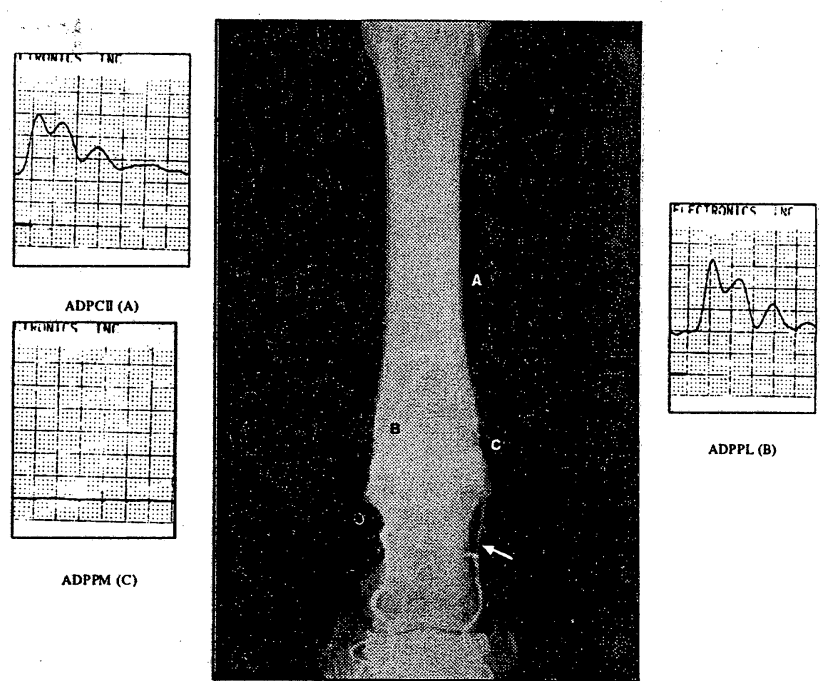

Figura 5: Arteriografia e Doppler Ultra-Som do caso 14. A arteriografia foi obtida na posição latero-medial (LM) e os registros do Doppler UltraSom nos locais indicados pelas letras. Observa-se preenchimento de todo o trajeto das artérias digitais e traçados com ondas de velocidade. $\mathrm{A}=$ artéria digital palmar comum $\mathrm{II} ; \mathrm{B}=$ artéria digital palmar própria lateral; $\mathrm{C}=$ artéria digital palmar própria medial.

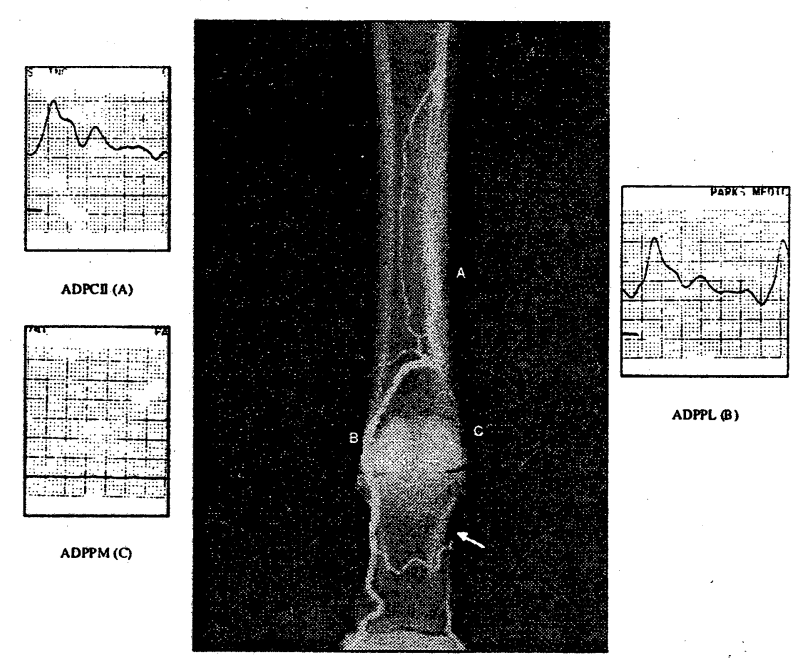

Figura 6: Arteriografia e Doppler Ultra-Som do caso 18. A arteriografia foi obtida na posição dorso-palmar (DPa) e os registros do Doppler UitraSom nos locais indicados pelas letras. Observa-se preenchimento de todo o trajeto das artérias digitais e traçados com ondas de velocidade. $\mathrm{A}=$ artéria digital palmar comum II; $\mathrm{B}=$ artéria digital palmar própria lateral; $\mathbf{C}=$ artéria digital palmar própria medial.

contínuos. Acreditamos que a etiologia das obstruções evidenciadas no nosso estudo possa estar relacionada à atividade atlética dos animais (equitação militar, salto, pólo) e à idade avançada dos mesmos, que contribuem para a ocorrência destas enfermidades.

A leitura da arteriografia do caso $n^{2} 4$ não foi conclusiva, pois - cateter saiu de sua posição intraluminal, durante a injeção de contraste, ocasionando um fraco preenchimento nos segmentos arteriais estudados. Esta saída do cateter resultou dos movimentos de flexão do membro apresentados pelo animal durante a realização da fase arteriográfica. Sobre a 
influência do contraste, Lessa (1993) atribui estes movimentos à sensação dolorosa ou a possíveis reações reflexas no membro durante a injeção de contraste. Este caso não foi utilizado nos cálculos estatísticos para correlação entre as técnicas.

\section{Doppler Ultra-sonográficos}

Em 56 segmentos arteriais onde o exame foi negativo (fluxo estava presente), foi possivel identificar os ruídos produzidos pelo fluxo arterial e observar no traçado tanto o sentido do fluxo como a morfologia das ondas. Estes resultados foram caracterizados no traçado pela presença inicialmente de ondas de velocidades crescentes e posteriormente decrescentes que se repetiam, formando segmentos de ondas com formas semelhantes a uma parábola (Figura 1).

Walker e Geiser (1986) identificaram este fenômeno na artéria metatársica dorsal III de cavalos e atribuem estes resultados ao caráter pulsátil e relativamente laminar do fluxo arterial. Acreditamos que esta forma das ondas de registro (crescentes e decrescentes) correspondam, respectivamente, às fases sistólica e diastólica do pulso arterial devido à capacidade do Doppler Ultra-Som captar as alterações de velocidade de fluxo nas artérias.

Sete exames foram positivos (não existia fluxo), onde não foram observados ruídos de fluxo arterial, sendo caracterizados por uma linha isoelétrica no papel de registro (Figura 2). Estes resultados apresentaram-se com a seguinte distribuição por segmento: seis na artéria digital palmar própria medial (casos no 1, 12, 16, 19, 20 e 21) e uma na artéria digital palmar própria lateral (caso $\mathrm{n} \times 7$ ).

Considerando o funcionamento do Doppler Ultra-Som, onde a velocidade de fluxo sangüíneo pode ser aferida devido à sua capacidade de captar as diferenças de freqüências entre as ondas ultra-sônicas emitidas por ele e as ondas refletidas pelos elementos figurados do sangue (efeito Doppler), as linhas isoelétricas encontradas nos traçados justificam-se pela ausência de reflexão de ondas sangüíneas devido às obstruções.

\section{Correlação dos resultados}

O número de segmentos arteriais examinados com resultados conclusivos perfizeram um total de 63 segmentos: 21 da artéria digital palmar comum II, 21 da artéria digital palmar própria medial e 21 da artéria digital palmar própria lateral.

Os resultados positivos e negativos do Doppler Ultra-Som foram comparados com os achados de presença e ausência de obstrução arterial das arteriografias, considerando os mesmos segmentos arteriais (Tabela 3 ).

Utilizando as incidências dos resultados de cada método, foi aplicado o teste Qui-quadrado (Tabela 4), e foram determina-. das a sensibilidade (probabilidade de que o resultado do exame do Doppler Ultra-Som seja positivo na presença da obstrução), a especificidade (probabilidade de que o resultado do exame do Doppler Ultra-Som seja negativo na ausência da obstrução), a incidência de falsos-positivos (registro de onda isoelétrica onde não existia obstrução) e a de falsosnegativos (registro de onda de fluxo onde existia obstrução) do método Doppler Ultra-Som.
Tabela 3: Comparação dos resultados Doppler Ultra-Som versus arteriografia

\begin{tabular}{|c|c|c|c|c|c|c|c|}
\hline \multirow[t]{2}{*}{ Caso } & \multirow[t]{2}{*}{ Membro } & \multicolumn{3}{|c|}{ Doppler } & \multicolumn{3}{|c|}{ Arteriografia } \\
\hline & & ADPC II & ADPPM & ADPPL & ADPC $\|$ & ADPPM & $\overline{A D P P L}$ \\
\hline 1 & TD & - & + & - & - & + & $\cdot$ \\
\hline 2 & TE & - & - & - & - & + & - \\
\hline 3 & TD & - & $\cdot$ & - & + & $\cdot$ & $\cdot$ \\
\hline 4 & TE & - & $\cdot$ & - & NC & NC & NC \\
\hline 5 & TD & - & - & - & - & $\cdot$ & - \\
\hline 6 & TE & - & - & - & - & - & - \\
\hline 7 & TD & - & - & - & - & - & - \\
\hline 8 & TE & $\cdot$ & $\cdot$ & - & - & . & - \\
\hline 9 & TD & - & - & - & - & - & - \\
\hline 10 & TE & - & - & - & - & - & - \\
\hline 11 & TD & - & - & - & - & - & - \\
\hline 12 & TE & $=$ & + & - & - & + & - \\
\hline 13 & TD & - & - & - & - & - & - \\
\hline 14 & TE & - & - & - & - & - & - \\
\hline 15 & TD & - & - & - & - & - & - \\
\hline 16 & TE & - & + & - & - & + & - \\
\hline 17 & TD & - & $\therefore$ & + & - & $\cdot$ & + \\
\hline 18 & TE & - & - & - & - & - & - \\
\hline 19 & TD & - & + & - & - & + & - \\
\hline 20 & TE & - & + & - & - & + & - \\
\hline 21 & TD & - & + & - & . & + & - \\
\hline 22 & TE & - & - & - & - & - & - \\
\hline
\end{tabular}

$T E=$ torácico esquerdo; $T D=$ torácico direito; + = presença de obstrução; - = ausência de obstrução; ADPC II = artéria digital palmar comum II ADPPM = artéria digital palmar própria medial; $A D P P L=$ artéria digital palmar própria lateral; NC = nāo conclusivo.

Tabela 4: Qui-quadrado aplicado aos resultados do Doppler Ultra-Som e da arteriografia, segundo Bellen (1987)

\begin{tabular}{cccc}
\hline \multirow{2}{*}{ Doppler } & \multicolumn{2}{c}{ Arteriografia } & \multirow{2}{*}{ Total } \\
\cline { 2 - 3 } & Presente & Ausente & \\
\hline Positivo & 7 & 0 & 7 \\
Negativo & 2 & 54 & 56 \\
\hline Total & 9 & 54 & 63 \\
\hline
\end{tabular}

O valor do cálculo do Qui-quadrado, para um grau de liberdade, foi igual a $\chi^{2}=47,25^{\star \star}(p<0,01)$, significativo ao nível de $1 \%$.

A correlação dos resultados quanto ao diagnóstico das obstruções arteriais demonstrou o Doppler Ultra-Som ter uma sensibilidade de $77,7 \%$ (indice de acerto na identificação das obstruções), pois dos nove segmentos arteriais digitais que se apresentavam obstruídos à arteriografia, sete foram positivos e dois foram negativos ao Doppler Ultra-Som (falsos negativos). Por outro lado, os 54 segmentos restantes, que se apresentavam negativos ao Doppler Ultra-Som, mostraram-se também com ausência de obstrução da luz arterial na arteriografia, alcançando uma especificidade de $100 \%$ (índice de acerto quando não existia a obstrução)

Segundo descrição de Schmidt e Duncan (1994), pelos altós indices de sensibilidade e especificidade alcançados neste 
estudo, o diagnóstico das obstruções está situado em uma área de alta probabilidade, pois mesmo não sendo necessariamente $100 \%$ certo, é tão provável, que nenhuma avaliação adicional é necessária. Da mesma forma, estes percentuais estão próximos daqueles relatados por Markel et al. (1995), cujos índices de sensibilidade chegaram a $85 \%$ e especificidade a $100 \%$ no diagnóstico da trombose venosa profunda.

Acreditamos que a incidência de dois casos falso-negativos $(23,3 \%)$, um na artéria digital palmar própria medial do caso no 2 e um na artéria digital palmar comum $\|$ do caso ํo 3 podem ser atribuídos aos seguintes fatores: no caso $n^{2} 2$, ter ocorrido a captação de ondas de fluxo em artérias da circulação colateral que se apresentava desenvolvida na região do segmento obstruído, corroborando com as afirmações de Gross et al. (1977) sobre a presença de vários ramos colaterais desenvolvidos em regiões de obstrução arterial. Da mesma forma, Coelho (1994) afirma que, em alguns casos, na região distal de uma obstrução, nem sempre podemos afirmar que a onda de velocidade é de uma artéria troncular ou de uma colateral. Por outro lado, atribuímos a ocorrência do falsonegativo no caso n 3 ao fato de terem sido necessárias três cateterizações na artéria digital palmar comum II para os procedimentos arteriográficos, fato que supomos ter levado a um espasmo ou a uma obstrução daquela artéria devido à excessiva manipulação ou à própria cateterização, induzindo a leitura de obstrução na arteriografia. Este fenômeno foi igualmente relatado por Ackerman et al. (1975) e Scott et al. (1976), resultando na ausência de preenchimento pelo meio de contraste na artéria digital palmar comum II.

\section{Referências bibliográficas}

ACKERMAN, N., GARNER, H.E., COFFMAN, J.R. et al. Angiographic appearance of the normal equine foot and alterations in chronic laminitis. J. Am. Vet. Med. Assoc., v. 166, n. 1, p. 58-62, 1975.

BELLEN, B.V. Métodos não-invasivos no estudo das doenças arteriais. In: MAFFEI, F.H.A. (Ed.). Doenças Vasculares Periféricas. Rio de Janeiro: MEDSI, 1987. p. 235-254.

BOTTI; P. Quadri arteriografici in alcune forme patologiche delle regioni metacarpo - falangea e digitale del cavallo. Atti della Societá Italiana delle Sienze Veterinarie, v. 37; p. 261-263, 1983.

BRUM, O. Doppler Ultra-Som: princípios básicos e aplicações clínicas. Rio de Janeiro: Livro Médico, 1986. 200 p.

CAMPOS, H. Estatística experimental não-paramétrica. 4. ed. Piracicaba: Escola Superior de Agricultura Luiz de Queirós, Universidade de São Paulo, [s. d.], 349 p.

COELHO, N.A., Troncos arteriais dos membros inferiores. Rev. Angiol. Cir. Vasc., v. 3, n. 1, p. 27-32, 1994.

COFFMAN, J.R., JOHNSON, J.H., GUFFY, M.M. et al. Hoof circulation in equine laminitis. J. Am. Vet. Med. Assoc., v. 156, n. 1, p. 76-83, 1970.

DUNLOP, G.R., SANTOS, R. Adductor-canal thrombosis. New. Engl. J. Med., v. 256, n. 13, p. 577-580, 1957.

FRICKER, C.H., RIEK, W., HUGELSHOFER, J. Oclusion of the digital arteries: a model for pathogenesis of navicular disease. Equine Vet. J., v. 14, n. 3, p. 203-207, 1982.

GROSS, W.S., VERTA, M.J., BELLEN, B.V. et al. Comparison of noninvasive diagnostic techniques in carotid artery occlusive disease. Surgery, v. 82, n. 2, p. 271-278, 1977.

HENRIQUES, E.O. Arteriografia digital palmar no eqüino em estação (Equus caballus). 1998. Tese (Mestrado) - Universidade Federal Rural do Rio de Janeiro (em fase de elaboração).
Embora não tenha ocorrido nenhum resultado falso-positivo pelo Doppler Ultra-Som, Morais (1988) afirma que estes falsos-positivos estão geralmente associados à calcificação intensa da parede arterial, dificultando a penetração das ondas de ultra-som na luz arterial, ou a erros de técnica de utilização do aparelho, seja no ajuste dos controles de registro gráfico do Doppler ou na falta de identificação da artéria. Brum (1986) relata que a ausência de registro de ondas de fluxo pode também ser atribuída a uma velocidade de fluxo menor que $6 \mathrm{~mm} / \mathrm{s}$ que não é captada pelo Doppler. Por outro lado, Miranda et al. (1980) afirmam que a acurácia diagnóstica do exame é proporcional à experiência do examinador e que esta pode influenciar nos resultados. Acreditamos que estes casos podem estar relacionados com a falta de identificação da artéria pelo transdutor, pois o exame Doppler Ultra-sonográfico é um estudo não-invasivo, no qual supomos que a anatomia da região e as resṕostas obtidas têm uma correlação direta, e, em alguns casos, isto não acontece.

\section{Conclusão}

O Doppler Ultra-Som é capaz de identificar as obstruções completas das artérias digitais do membro torácico no eqüino e de selecionar casos obstrutivos para o estudo pré-cirúrgico arteriográfico.

Os resultados estatísticos indicam ser o exame mais desejável para uma população de cavalos com alta incidência de lesões obstrutivas nas artérias digitais do membro torácico para evitar exames invasivos desnecessários.

HERSTCH, B. Zur arteriographie der zehe des pferds. Berl. Münch. Tierärztl. Wschr., v. 86, n. 24, p. 461-465, 1973.

KÖHLER, H. Endarteriitis obliterans der zehenarterien beim pferd. $Z$. Path., v. 62, p. 326-344, 1951.

LESSA, D.A.B. Técnicas de punção e cateterização para arteriografia palmarno cavalo (Equus caballus). 1993. 77 p. Tese (Mestrado) Faculdade de Medicina Veterinária - Universidade Federal Fluminense.

LESSA, D.A.B., SILVA, A.C., RESTUM, M.A.L. Estudo da via de acesso longitudinal para punção e cateterização da artéria digital palmar comum II no cavalo (Equus caballus) para fins arteriográficos. Rev. Bras. Ciênc. Vet., v. 4, n. 3, p. 121-125, 1997.

LITZKE, L.F. Der einsatz der angiographie bei der lahmheitsdiagnostik. Mh. Vet. Med., v. 41, n. 10, p. 347-348, 1986.

MARKEL, A., WEICH, Y., GAITINI, D. Doppler ultrasound in the diagnosis of venous trombosis. Angiology, v. 46, n. 1, p. 65-73, 1995.

MIRANDA Jr., F., BURIHAN, E. Doppler vascular. In: DOLNIKOFF, M. (Ed.) Ultra-Sonografia. São Paulo: Cip-Brasil, 1980. p. 449-474.

MORAIS, I.N. Propedêutica vascular. 2. ed. São Paulo: Sarvier, 1988. $240 \mathrm{p}$.

PANICO, M.D.B. O valor da fluxometria ultra-sônica nas doenças oclusivas das carótidas. Rev. Angiol. Cir. Vas., v. 4, n. 1, p. 7-16, 1995.

SCHMIDT, M.I., DUNCAN, B.B. O método epidemiológico na conduta e na pesquisa clínica. In: ROUQUAYROL, M.Z. (Ed.). Epidemiologia e saúde. 4. ed. Rio de Janeiro: MEDSI, 1994. p. 200-204.

SCOTT, E.A., THRALL, D.E., SANDLER, G.A. Angiography of equine metacarpus and phalanges: alterations with medial palmar artery and medial palmar digital artery ligation. Amer. J. Vet. Res., v. 37, n. 8, p. 869-873, 1976. 
SILVA, A.C. Arteriografia Digital Palmar Comum II no Cavalo (Equus caballus). 1988. 64 p. Tese (Mestrado) - Faculdade de Medicina Veterinária - Universidade Federal Fluminense.

SILVA, A.C. Estudo do padrão da distribuição anátomo-radiográfico dos ramos colaterais palmares do arco terminal no eqüino sem raça definida (Equus caballus). 1998. 120 p. Tese (Doutorado) Faculdade de Medicina Veterinária - Universidade de São Paulo.

SILVA, A.C., PENA, N.D., DA COSTA, L.A.P. et al. Diagnóstico de oclusão das artérias digitais palmares próprias no cavalo (Equus caballus). In: CONGRESSO BRASILEIRO DE MEDICINA VETERINÁRIA, 21., 1995, Salvador, Anais... p. 92.
STANEK, C.H., GIRTLER, D. Technik der arteriographie der pferdezehe am stehenden tier. Berl. Münch Tierärztl. Wschr., v. 95, n. 5, p. 8791, 1982.

TURNER, T.A., PUROHIT, R.C., FESSLER, J.T. Themography: a review in equine medicine. Compend. Contin. Educ. Pract. Vet., v. 8, n. 11, p. 855-861, 1986.

WALKER, M., GEISER, D. Effects of acetylpromazine on the hemodynamics of the equine metatarsal artery, as determined by two-dimensional real-time and pulsed doppler ultrasonography. Am. J. Vet. Res., v. 47, n. 5, p. 1075-1078, 1986. 